\title{
Parametric vibration analysis and validation for a novel portable hexapod machine tool attached to surfaces with unequal stiffness
}

\author{
Nan Ma1,2, Xin Dong*2, David Palmer², Josue Camacho Arreguin², Zhirong Liao², Mingfeng Wang², Dragos Axinte ${ }^{2,3}$ \\ ${ }^{1}$ Robotics Institute, Beihang University, Beijing, 100191, China \\ 2 Department of Mechanical, Materials and Manufacturing Engineering, School of Engineering, University of Nottingham, NG7 2RD, \\ United Kingdom \\ ${ }^{3}$ Department of Mechanical, Materials and Manufacturing Engineering, School of Engineering, University of Nottingham Ningbo \\ China, 315100, China;
}

*Corresponding author: xin.dong@nottingham.ac.uk

\begin{abstract}
The Free-Leg Hexapod (Free-Hex) machine tool is an advancement from the conventional Stewart platform, which has the fixed base platform removed to enable the limbs to be attached to a wider range of surfaces (e.g. non-flat and curved). However, in some scenarios (e.g. in-situ repair of industrial installations), the limbs of the Free-Hex need to be attached to the surfaces with unequal stiffness, which brings the challenge of predicting the dynamics of the system for conducting machining operations under dynamically stable conditions. In this paper, after introducing the attachment stiffness (i.e. feet attached to the environment with different materials) in the conventional dynamic model of the parallel manipulator, the dynamic behavior of the Free-Hex machine tools devoted to the insitu operation environments was studied. Then, the experimental validation was conducted to prove the dynamic model developed in this paper. It was found that the errors of the proposed model are under $6 \%$ (i.e. $5.1 \%$ at symmetrical limb configuration and $5.8 \%$ at the arbitrary configuration) when the limbs are attached to the surfaces with unequal stiffness. Further, by applying the validated model, the dynamic performance of the Free-Hex with a wider range of attachment stiffness was analyzed. Overall, it was found that the attachment stiffness has a remarkable influence on the natural frequencies of the machine tool (e.g. the frequency of mode 4 at the symmetrical configuration is increased by $36.8 \%$ when the attachment stiffness of one limb changes from 0 to $1 \mathrm{e}+12 \mathrm{~N} / \mathrm{m}$ ). Thus, the work discussed in this paper can be utilized to avoid the dynamically unstable configurations of parallel kinematic machine tools (e.g. Free-Hex) when mounting on the surface with unequal stiffness in the in-situ operation environments.
\end{abstract}

Keywords: dynamic analysis, parallel kinematics machine tool, parametric vibration; unequal attachment stiffness

\section{Introduction}

To shorten the repair time of large installations (e.g. ship engines, off-shore platforms), portable/movable machine tools are increasingly utilized for in-situ operations, instead of disassembling the system and transporting them to the maintenance center $[1,2,3]$. This approach brings the advantages of saving transportation costs and reducing maintenance time. However, the in-situ maintenance requires the reinstallation, reconfiguration and recalibration of the machine tools against the features of the targets, which is of key importance for maintaining the original high accuracy and dynamic characteristics of the machine tools (e.g. modal frequencies and mode shape) as they have been originally designed. In addition, since the setup (i.e. foot location, attachment stiffness and spindle position) of the machine tools depends on the working environments (e.g. physical obstacles to access the targeted 
feature, the surfaces where the machine tools are attached), the dynamic characteristics, remarkably affect the machining stability, have to be analysed before setting up the machine tool for in-situ repair of industrial installations.

As a novel portable machine tool for in-situ repair and maintenance in challenging environments (e.g. power stations, off-shore platforms), the Free-Hex was developed by the inspiration of the conventional Stewart platform, which has been demonstrated as a precision machine tool $[4,5,6]$. With the base platform removed, the Free-Hex is able to be attached to a wider range of surfaces, e.g. flat and non-flat (Figure 1), compared with the conventional fixed-base machine tool. As the space for the limb attachments is often constrained when the machine tool utilized for in-situ operations compared with lab/factory environments, the limbs usually have to be clamped to the surfaces with unequal stiffness. The machining abilities of the Free-Hex in lab/factory environments (i.e. the attachment stiffness of all the feet are regarded as infinite) have been discussed in detail [7,8]. However, to the best of authors' knowledge, no research has been conducted for analyzing the dynamics of parallel kinematics machine tools when attached to unequal stiffness surface. Hence, the new application scenario for parallel kinematics machine tools (e.g. Free-Hex) is studied in this paper, which locates the system on the surface with unequal stiffness when doing in-situ intervention tasks, requiring an innovative dynamic model by considering extra parameters (e.g. attachment stiffness) for predicting its dynamic behavior.

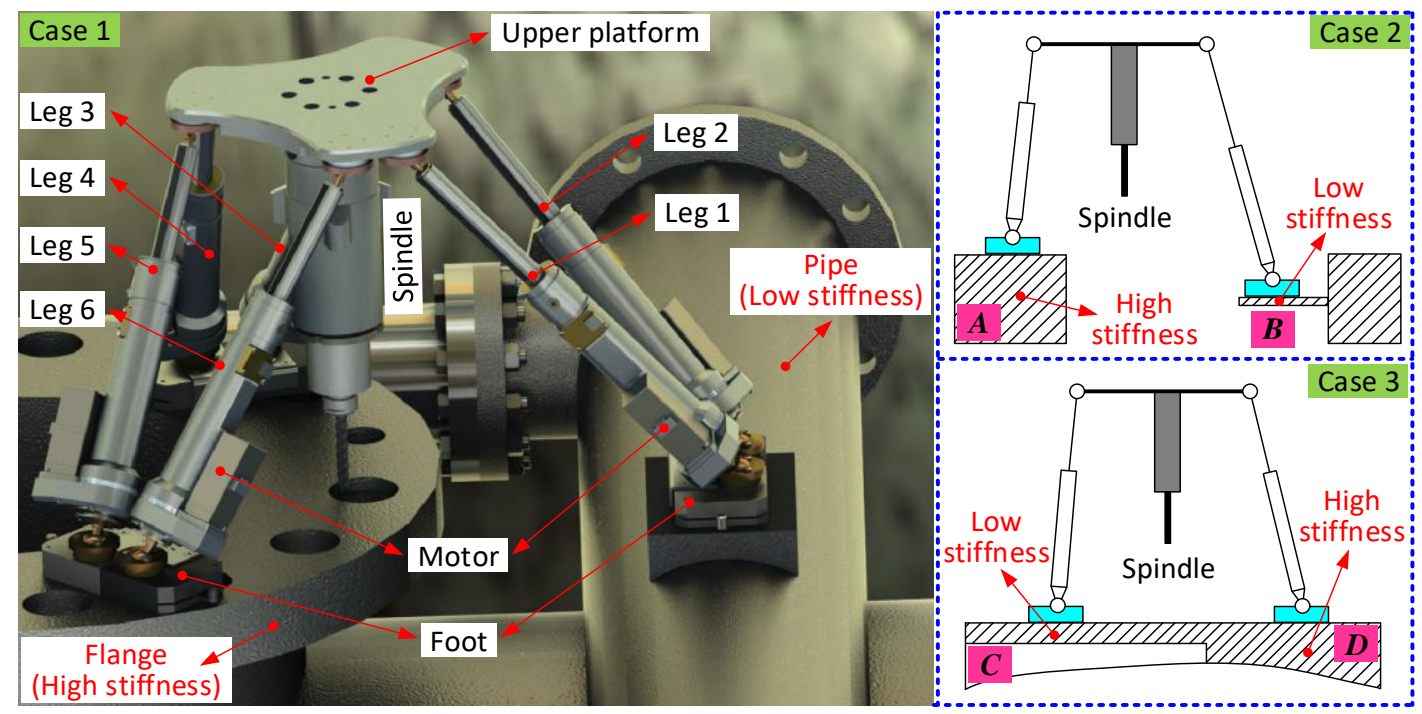

Figure 1. The examples of in-situ intervention in industrial installations: case 1 - valve repair, when Free-Hex is attached to different components with unequal stiffness (e.g. flange - high stiffness; pipe - low stiffness); case 2- Free-Hex is located on different parts (solid $(\boldsymbol{A})$ - high stiffness; cantilever beam $(\boldsymbol{B})$ - low stiffness); case 3 - Free-Hex is located on one piece with different wall thickness (shell $(\boldsymbol{C})$ - low stiffness; solid $(\boldsymbol{D})$ - high stiffness)

Regarding parallel kinematics machine tools, the identification of the dynamic behavior $[9,10,11]$ can be easily conducted by experiments (e.g. impact testing) supported by dynamic models when the machines are placed in an open workspace (e.g. lab/factory) and generally attached to the surfaces with infinite stiffness. Up to now, a considerable effort has been put on the study of the dynamic characters of parallel kinematics machine tools in unconstrained environments. For example, the dynamics model of a large 6 DoF Stewart platform-based machine tool was experimentally developed by assembling the dynamic model of each substructure to predict the natural frequency of the entire system [12]. As a desk-top Stewart platform-based machine tool, the dynamic model of the system was established by considering joint connections and structure configurations [13]. Further, the external loads with different loading frequencies have been also considered in the dynamic modelling of parallel kinematics machining tools, and the corresponding dynamic stability index was proposed and validated for improving the 
dynamic characteristics to enable the improvement of machining performance [14]. The dynamic equation of a kind of high-speed parallel robot was established to evaluate the performance characteristic variation during the working [15]. The dynamic response analysis was performed on a flexible spatial parallel mechanism to identify the chaos to improve the performance of the system [16]. It can be found that most of the previous researches consider that the feet of the parallel kinematic machine tool are fixed on the ground/workpiece in unconstrained spaces and the attachment stiffness is regarded as infinite $[17,18]$. This is not surprising since most of the hexapod machine tools have a fixed base-platform. However, these existing models cannot be applied for in-situ repair, since the attachment stiffness cannot be considered as infinite over the whole workpiece as the real industrial setups where such in-situ repairs are made are far from "ideal" conditions.

Further, the experimental modal analysis (EMA) approach involves numerous repetitions in lab/factory working environments [19], which are time-consuming and complex. However, when utilizing the portable machine tools for in-situ repair, the attachment stiffness varies when the machine attached to different locations, requiring even more measurements. Therefore, it is highly desirable to develop a novel parametric dynamic model to accurately predict the natural frequency of the machine tool for in-situ interventions, which can quickly obtain the dynamic characteristics of the system to improve its machining accuracy and service time.

In this paper, a novel parametric dynamic model of the Free-Hex machine tool, which considers the in-situ conditions (i.e. unequal attachment stiffness over the entire workpiece), is developed to solve the abovementioned challenges. Firstly, the kinematics (i.e. position, velocity and acceleration) of the Free-Hex machine tool are calculated in chapter 2, which are utilized to establish the kinetic and potential energy of the system. Then, a new Lagrange dynamic equation, which considers the leg masses and attachment stiffness of limbs are established to obtain the natural frequencies in chapter 3 . Then, several case studies were implemented to analyze the influence of the leg's masses and the attachment stiffness on the natural frequencies under two classical configurations (i.e. symmetrical and arbitrary leg layout) by the proposed model, which were validated by simulation. In order to evaluate the dynamic model, the Free-Hex machine tool was attached to a mechanism able to adjust its stiffness for acquiring its natural frequencies when the feet are on surfaces with unequal stiffness in chapter 5. Finally, the conclusion is presented in chapter 6 .

\section{Kinematics modelling of Free-Hex machine tool}

In order to support the dynamic modelling of the Free-Hex machine tool, the kinematic model is developed for calculating the key parameters of the dynamic equation and mapping the structures of the Free-Hex machine in different configurations. As the legs are regarded as two separate parts of a telescopic structure, the corresponding kinematic parameters of their centroids are calculated to evaluate the equivalent kinetic and elastic energies for the dynamic equation of the system.

\subsection{Coordinate system definition}

Let us consider the Free-Hex with its feet fixed on surfaces with unequal stiffness (feet 1 to 2 with stiffness B, and feet 3 to 6 with stiffness $\mathrm{A}$ ) and its coordinate systems are defined to be $\{M\}$ for the machine tool and $\{U\}$ the for the upper platform (Figure 2). 


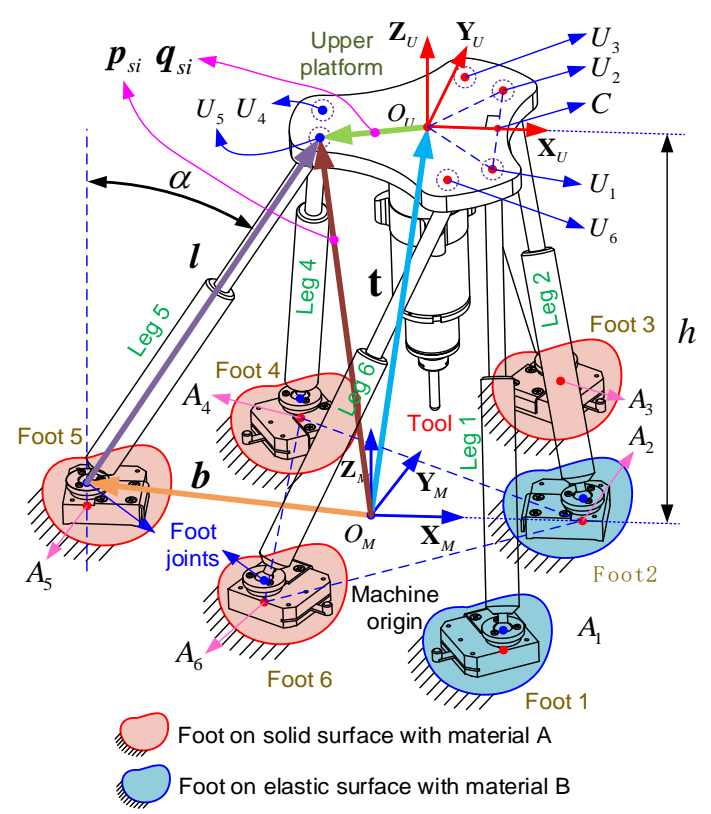

Figure 2. Free-Hex machine tool with feet fixed on surfaces with unequal stiffness with its coordinate system and key points definition

Global coordinate system $\{M\}$ : The origin is located at the geometry centre of the triangle formed by the foot centers $A_{2}, A_{4}$ and $A_{6}$. The positive direction $\boldsymbol{X}_{M}$ is defined from the origin to the foot center point $A_{2}$. The positive $\boldsymbol{Z}_{M}$ direction is vertical with the triangle. $\boldsymbol{Y}_{M}$ direction is defined using the right-hand rule.

Upper platform coordinate system $\{U\}$ : The origin is located at the center of the upper platform. The positive direction $\boldsymbol{X}_{U}$ is defined from the origin to the center point between the $U_{1}$ and $U_{2}$, the positive direction $\boldsymbol{Z}_{U}$ is same with $\boldsymbol{Z}_{M}$ in the initial time, the positive direction of $\boldsymbol{Y}_{U}$ is defined using the right-hand rule.

\subsection{Kinematic modelling}

In order to calculate the velocity and acceleration at the key points (e.g. spherical joints, centroids of the struts), the kinematic modelling of the Free-Hex is developed, which was used to establish the dynamic model of the FreeHex in Chapter 3. To properly represent the structure of the Free-Hex in a simplified form, the legs are considered as two separate parts (i.e. linear motor and linear shaft), which are connected to the lower feet and upper platform. It renders the dynamic characteristics at the centroids of two parts are different from the existing models, so it needs to be calculated separately.

The closed-loop vector of one leg in the Free-Hex is shown in Figure 2. With given position and orientation of the upper platform, the position vector of the spherical joint in the upper platform can be written as (Eq.(1)):

$$
\boldsymbol{p}_{s i}=\boldsymbol{t}+\mathbf{R} \boldsymbol{q}_{s i}
$$

Where:

$\boldsymbol{t}$ is the position vector of the upper platform with respect to $\{M\}$;

$\boldsymbol{R}$ is the rotation matrix of the upper platform with respect to $\{M\}$;

$\boldsymbol{p}_{s i}$ is the position vector $\left(O_{M} U_{i}\right)$ of the upper platform spherical joint $\mathrm{U}_{i}$ in $\{M\}$;

$\boldsymbol{q}_{s i}$ is the position vector $\left(O_{U} U_{i}\right)$ of the upper platform spherical joint $\mathrm{U}_{i}$ in $\{U\}$;

Taking the derivative of Eq.(1), the velocity vector, $\dot{\boldsymbol{p}}_{s i}$, of the spherical joint point can be written as [20]:

$$
\dot{\boldsymbol{p}}_{s i}=\dot{\boldsymbol{t}}+\boldsymbol{\omega} \times \mathbf{R} \boldsymbol{q}_{s i}=\dot{\boldsymbol{t}}+\mathbf{R} \tilde{\boldsymbol{q}}_{s i}^{T} \mathbf{R}^{T} \boldsymbol{\omega}=\left[\begin{array}{ll}
\mathbf{I}_{3 \times 3} & \mathbf{R} \tilde{\boldsymbol{q}}_{s i}^{T} \mathbf{R}^{T}
\end{array}\right]\left[\begin{array}{c}
\dot{\boldsymbol{t}} \\
\boldsymbol{\omega}
\end{array}\right]=\left[\begin{array}{ll}
\mathbf{I}_{3 \times 3} & \mathbf{R} \tilde{\boldsymbol{q}}_{s i}^{T} \mathbf{R}^{T}
\end{array}\right] \dot{\boldsymbol{q}}
$$


Where:

$\dot{\boldsymbol{t}}$ is the generalized velocity of the origin of the upper platform;

$\boldsymbol{\omega}$ is the generalized rotation velocity of the upper platform;

$\widetilde{\boldsymbol{q}}_{s i}$ is the skew-symmetric matrix of $\boldsymbol{q}_{s i}$;

$\dot{\boldsymbol{q}}$ is the generalized velocity of the upper platform with respect to $\{M\}$;

The rotation angular velocity $\boldsymbol{\omega}_{l i}$ of the leg relative to the lower spherical joint point (Figure 3 ) can be written as:

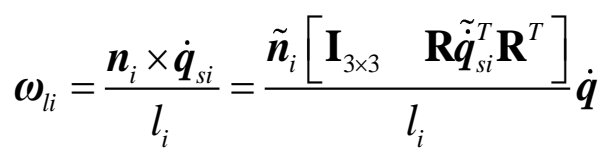

Where:

$\boldsymbol{n}_{i}$ is the unit vector along the axis of the leg;

$\widetilde{\boldsymbol{n}}_{i}$ is the skew-symmetric matrix of the unit vector $\boldsymbol{n}_{i}$;

$l_{i}$ is the length of the leg;

Each leg of the Free-Hex is composed of two parts, an upper part (i.e. a linear shaft) and a lower part (i.e. a linear motor) respectively. The distances between the mass center of the upper and lower parts to the joint center points $U_{i}$ and $A_{i}$ are $l_{t}$ and $l_{h}$ (Figure 3). The upper part gravity is $m_{t} \boldsymbol{g}$, and the direction is vertical downward. The gravity vector of the lower part is denoted as $m_{b} \boldsymbol{g}$, and the direction is also vertical downward, as shown in Figure 3.

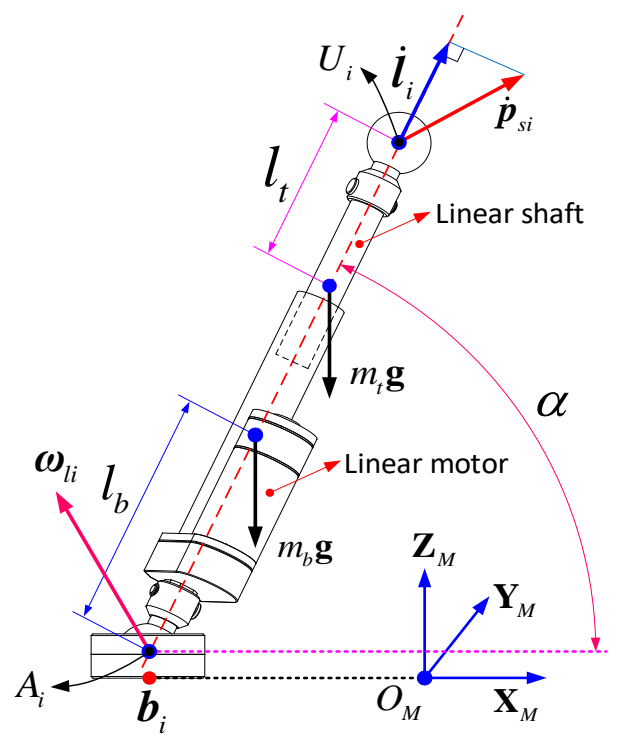

Figure 3. Kinematic model of one single leg

The lower and upper parts of a leg share the same angular velocity $\boldsymbol{\omega}_{l i}$. Then, the generalized motion velocity [21] of lower and upper parts can be obtained by using Eq.(2).

$$
\begin{gathered}
\boldsymbol{v}_{t i}=\dot{\boldsymbol{q}}_{s i}+\boldsymbol{\omega}_{l i} \times\left(-\boldsymbol{l}_{t i} \boldsymbol{n}_{i}\right)=\left(\mathbf{I}_{3 \times 3}+\frac{l_{t} \tilde{\boldsymbol{n}}_{i}^{2}}{l_{i}}\right) \dot{\boldsymbol{p}}_{s i}=\left(\mathbf{I}_{3 \times 3}+\frac{l_{t} \tilde{\boldsymbol{n}}_{i}^{2}}{l_{i}}\right) \cdot\left[\begin{array}{ll}
\mathbf{I}_{3 \times 3} & \mathbf{R} \tilde{\boldsymbol{p}}_{s i}^{T} \mathbf{R}^{T}
\end{array}\right] \dot{\boldsymbol{q}} \\
\boldsymbol{v}_{b i}=\boldsymbol{\omega}_{l i} \times l_{b} \boldsymbol{n}_{i}=\left(\frac{l_{b} \tilde{\boldsymbol{n}}_{i}^{T} \tilde{\boldsymbol{n}}}{l_{i}}\right) \dot{\boldsymbol{p}}_{s i}=\left(\mathbf{I}_{3 \times 3}+\frac{l_{b} \tilde{\boldsymbol{n}}_{i}^{2}}{l_{i}}\right) \cdot\left[\begin{array}{ll}
\mathbf{I}_{3 \times 3} & \left.\mathbf{R} \tilde{\boldsymbol{p}}_{s i}^{T} \mathbf{R}^{T}\right] \dot{\boldsymbol{q}}
\end{array}\right.
\end{gathered}
$$


Where:

$v_{t i}$ is the generalized velocity of upper part mass center in $i$-th leg;

$\boldsymbol{v}_{b i}$ is the generalized velocity of lower part mass center in $i$-th leg;

$\widetilde{\boldsymbol{n}}_{i}$ is the skew-symmetric matrix of unit vector of $i$-th leg;

$\widetilde{\boldsymbol{p}}_{s i}$ is the skew-symmetric matrix of coordinates vector of the $i$-th spherical joint in $\{U\}$;

As the generalized angle velocities and motion velocities of the two separate parts of a leg have been calculated in Eq.(4) and Eq.(5), the kinetic energy of the six legs can be determined and added to the total energy of the system to further establish the dynamic equation of Free-Hex by using the Lagrange method.

\section{Dynamic modelling of Free-Hex machine tool}

The stiffness of the contacting surface is considered for the first time in establishing the dynamic equations of Free-Hex machine. Then the dynamic characteristics affected by the attachment stiffness, as well as the masses, damping and stiffness of the legs $[22,23,24]$, can be analyzed separately to explore the performance variation at arbitrary machine configurations, which can help to forecast the machine performance and guarantee the quality in the preparation stage [25].

Further, the dynamic model developed in this paper can be extended to be applied to a family of parallel kinematics machines (e.g. 3RPS and 3UPU), once their feet are not constrained by based platform, they also can be utilized as portable machine tools for repair and maintenance on complex workpieces surfaces.

\subsection{Attachment stiffness consideration}

As commented, the Free-Hex is designed for in-situ repair and maintenance in extreme environments and this requires, in some instances, the limbs have to be attached to surfaces with unequal stiffness for conducting machining tasks (Figure 1). This scenario has not been considered in the previous researches, which impacts the natural frequencies of the Free-Hex machine tool, leading to the changes in the dynamic characteristics of the system, and also affects the machining performance of the system as a result.

For modelling the attachment stiffness, a generic situation is shown as Figure 4, which assumes a foot attached on a surface with finite stiffness $\left(k_{s}\right)$ [26], and the angle between the axis of the leg and the normal direction of the surface to be $\alpha$, as seen in Figure 4.

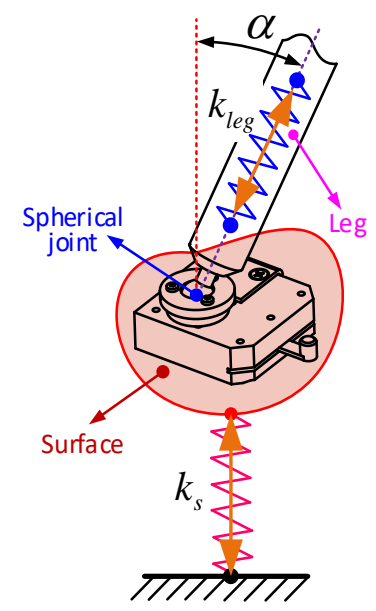

Figure 4. Equivalent stiffness calculation with the consideration of the attachment stiffness

The attachments stiffness is then considered to get the equivalent stiffness of a leg in Free-Hex for establishing 
the dynamic equation, and the equivalent stiffness can be expressed as:

$$
K=\frac{1}{\frac{1}{k_{s} / \sin (\alpha)}+\frac{1}{k_{l e g}}}
$$

Where:

$K$ is the overall stiffness of support including the stiffness of the leg and attachment (i.e. contact surface);

$k_{s}$ is the stiffness of the contacting surface; $k_{\text {leg }}$ is the stiffness of the leg;

$\alpha$ is the angle of the leg vector with the normal direction of the attachment.

From Eq.(6), it can be found that the attachment stiffness and angle $\alpha$ can influence the equivalent stiffness of one leg, which changes the dynamic characteristics of the Free-Hex as a result. When a foot is located on the surface with infinite stiffness (e.g. concrete ground and thick metal surface), the equivalent overall stiffness of the support (including the leg and the attachment) can be approximated as the leg stiffness; while once the foot is located on the surface with finite stiffness (e.g. thin pipe), the equivalent overall stiffness of the support can be varied by the attachment stiffness and the angle between the normal direction of the surface and the leg axis. The equivalent stiffness model of one leg will be utilized in the dynamic equation presented in this paper to analyze the dynamic characteristics of the Free-Hex with different configurations and surface stiffness.

\subsection{Dynamic equation of the Free-Hex}

A new dynamic equation of the Free-Hex is established in this section to analyze the dynamic characteristics by considering unequal attachment stiffness among the six feet, leading to a wider range of applications of the Free-Hex in in-situ environment with better performances; it also can be utilized to a derived family of parallel kinematics machines (e.g. 3RPS and 3UPU), which have removed base platforms [27,28].

The free vibration equation can be established to get the dynamic characteristics of Free-Hex, which is expressed in the following form:

$$
\boldsymbol{M} \ddot{\boldsymbol{q}}+\boldsymbol{C} \dot{\boldsymbol{q}}+\boldsymbol{K} \boldsymbol{q}=\boldsymbol{O}
$$

Where, $\boldsymbol{M}$ is the mass matrix, $\boldsymbol{C}$ is the damping matrix and $\boldsymbol{K}$ is the stiffness matrix of the Free-Hex machine. $\ddot{\boldsymbol{q}}, \dot{\boldsymbol{q}}$ and $\boldsymbol{q}$ are the generalized acceleration, velocity and displacement of the system.

The mass matrix can be obtained by differentiating the total kinetic energy to the generalized velocity of the system. The total kinetic energy of the system, $T$, includes the upper platform's kinetic energy $T_{u p p}$ and six legs' kinetic energy $T_{l e g}$. The upper platform's kinetic energy can be calculated easily, which is composed of the translational energy $T_{\text {move }}$ and rotational energy $T_{\text {rot }}$ of the upper platform [21], as shown.

$$
\begin{aligned}
& T_{u p p}=T_{\text {move }}+T_{r o t}=\frac{1}{2}\left(\dot{t}_{x}^{2}+\dot{t}_{y}^{2}+\dot{t}_{z}^{2}\right) m+\frac{1}{2} \boldsymbol{I}_{x x}(\dot{\alpha}-\dot{\gamma} \mathrm{s} \beta)^{2} \\
& +\frac{1}{2} \boldsymbol{I}_{y y}(\dot{\beta} c \alpha+\dot{\gamma} c \beta s \alpha)^{2}+\frac{1}{2} \boldsymbol{I}_{z z}(-\dot{\beta} s \alpha+\dot{\gamma} c \beta c \alpha)^{2}
\end{aligned}
$$

Where:

$\dot{t}_{x}, \dot{t}_{y}$ and $\dot{t}_{z}$ are the velocity components of the upper platform in $\{M\}$;

$\boldsymbol{I}_{x x}, \boldsymbol{I}_{y y}$ and $\boldsymbol{I}_{z z}$ are inertia tensors matrix of the upper platform in $\{M\}$;

$\alpha, \beta$ and $\gamma$ are Euler angles around $\boldsymbol{X}, \boldsymbol{Y}$ and $\boldsymbol{Z}$ axes of the upper platform in $\{U\}$;

$\dot{\alpha}, \dot{\beta}$ and $\dot{\gamma}$ are the Euler angular velocities of the upper platform in $\{U\}$; 
The total kinetic energy of the leg contains the translational energy and rotational energy. The leg is constructed by two separate parts (i.e. linear motor and linear shaft respectively) with the masses concentrated in their centroids. Since the lower part (linear motor) is connected to the lower spherical joint and can only realize rotational motion, and the upper part (linear shaft) is connected to the upper spherical joint is able to perform rotational and translational motion at the same time. Then the total energy can be written as [20]:

$$
T_{\text {leg }}=\frac{1}{2} \sum_{i=1}^{6} \boldsymbol{v}_{t i}^{T} m_{t} \boldsymbol{v}_{t i}+\frac{1}{2} \sum_{i=1}^{6} \boldsymbol{\omega}_{l i}^{T}\left(\boldsymbol{I}_{t}+\boldsymbol{I}_{b}\right) \boldsymbol{\omega}_{l i}
$$

Substituting Eq.(3), (4) and (5) into Eq.(9), the parametric kinetic energy of the legs can be obtained as Eq.(10).

$$
T_{\text {leg }}=\frac{1}{2} \sum_{i=1}^{6} \dot{\boldsymbol{q}}^{T}\left(\boldsymbol{M}_{1 i}^{T} m_{t} \boldsymbol{M}_{1 i}+\boldsymbol{M}_{2 i}^{T}\left(\boldsymbol{I}_{t}+\boldsymbol{I}_{b}\right) \boldsymbol{M}_{2 i}\right) \dot{\boldsymbol{q}}
$$

Where,

$$
\begin{gathered}
\boldsymbol{M}_{1 i}=\left(\mathbf{I}_{3 \times 3}+\frac{l_{t} \tilde{\boldsymbol{n}}_{i}^{2}}{l_{i}}\right) \cdot\left[\begin{array}{ll}
\mathbf{I}_{3 \times 3} & \mathbf{R} \tilde{\boldsymbol{p}}_{s i}^{T} \mathbf{R}^{T}
\end{array}\right] \\
\boldsymbol{M}_{2 i}=\frac{\tilde{\boldsymbol{n}}_{i}\left[\begin{array}{ll}
\mathbf{I}_{3 \times 3} & \mathbf{R} \tilde{\boldsymbol{p}}_{s i}^{T} \mathbf{R}^{T}
\end{array}\right]}{l_{i}}
\end{gathered}
$$

The inertia matrix $\boldsymbol{M}$ of the Free-Hex can be obtained by differentiating the total energy with respect to the generalized velocity vector $\dot{\boldsymbol{q}}$, and then substituting Eq.(8) and (10) into Eq.(13):

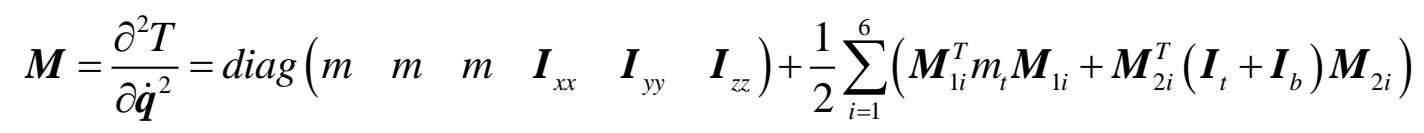

The stiffness matrix $\boldsymbol{K}$ of the Free-Hex can be obtained by differentiating the potential energy with respect to the position vector $\boldsymbol{q}$ :

$$
\boldsymbol{K}=\frac{\partial^{2} V}{\partial \boldsymbol{q}^{2}}=\frac{\partial^{2}}{\partial \boldsymbol{q}^{2}}\left(\frac{1}{2} \sum_{i=1}^{6} k_{i} \Delta l_{i}^{2}\right)=\sum_{i=1}^{6}\left(k_{i}\left(\frac{\partial l_{i}}{\partial \boldsymbol{q}}\right)^{T} \frac{\partial l_{i}}{\partial \boldsymbol{q}}+k_{i} \Delta l_{i} \frac{\partial^{2} l_{i}}{\partial \boldsymbol{q}^{2}}\right)=\boldsymbol{J}^{T} \operatorname{diag}\left(K_{1}, \cdots, K_{6}\right) \boldsymbol{J}
$$

Where, $K_{1}, \ldots, K_{6}$ are the equivalent stiffness coefficient of each leg. $\boldsymbol{J}$ is the Jacobi matrix of the Free-Hex, as shown in Eq.(15).

$$
\boldsymbol{J}=\left[\begin{array}{ccc}
\boldsymbol{n}_{1}^{T} & \cdots & \left(\mathbf{R} \boldsymbol{p}_{s 1} \times \boldsymbol{n}_{1}\right)^{T} \\
\vdots & \ddots & \vdots \\
\boldsymbol{n}_{6}^{T} & \cdots & \left(\mathbf{R} \boldsymbol{p}_{s 6} \times \boldsymbol{n}_{6}\right)^{T}
\end{array}\right]
$$

As the damping matrix, $\boldsymbol{C}$, is proven to be proportional to the stiffness, $\boldsymbol{K}$, it can be expressed as [28]:

$$
C=\boldsymbol{J}^{T} \operatorname{diag}\left(C_{1}, \cdots, C_{6}\right) \boldsymbol{J}=\boldsymbol{J}^{T} \operatorname{diag}\left(\lambda K_{1}, \cdots, \lambda K_{6}\right) \boldsymbol{J}=\lambda \boldsymbol{K}
$$

Where, $\lambda$, is the coefficient of proportionality between stiffness and damping of leg.

In this paper, as the stiffness coefficients of the legs are regarded as constant under different system configurations, the equivalent stiffness of the whole support (i.e. the leg and contacting surface) varies when the attachment stiffness changes. Then the dynamic characteristics of the Free-Hex are calculated and tested to investigate 
the vibration variation of the machine, as well as to validate the theoretical derivation presented in this paper. As the vibration equation is expressed parametrically by the kinematic and dynamic characteristics of the system, it is easy to find the contribution of different factors on the vibration of the system.

Using the coefficient matrix calculated in Eq.(13) and (14), the undamped dynamic vibration characteristic equation of the Free-Hex can be established, and the natural frequencies can also be obtained through calculating the eigenvalue and eigenvectors using Eq.(17).

$$
\left(\boldsymbol{M}^{-1} \boldsymbol{K}-\omega^{2} \boldsymbol{I}_{6 \times 6}\right) \boldsymbol{X}=\boldsymbol{0}
$$

Where, $\omega$ is the undamped natural frequency of the system corresponding to the specific eigenvector $\boldsymbol{X}$.

Based on Eq.(17), the dynamic characteristics of Free-Hex can be easily forecasted before the real setup of FreeHex in working environments. Moreover, the natural frequencies of the Free-Hex in six directions can also be calculated by the use of Eq.(17), which helps to acquire the natural frequencies and avoid resonances. In addition, as the attachment stiffness is also considered in the dynamic model, the more accurate dynamic characteristics and vibration modes of the Free-Hex can be predicted when it is used in the intervention site and the feet must be attached on surfaces with different boundary conditions (i.e. different materials and different structures, Figure 1).

There is now a possibility to determine the dynamic characteristics of Free-Hex in two scenarios:

1. Feet randomly distributed but on the surfaces with equal stiffness: in this case, the attachment stiffness is regarded as equal in Eq.(6), and then the natural frequencies can be calculated using the Eq.(17).

2. Feet randomly distributed but on the surfaces with unequal stiffness: in this case, the attachment stiffness is different among the feet, and the attachment stiffness should be allocated with corresponding different values in Eq.(6). Then, the natural frequencies can be calculated using Eq.(17).

Further, the effect of attachment stiffness on natural frequencies were studied in two aspects in the following sections: a) the experimental method was utilized to validate the results obtained from the theoretical calculation and simulation; b) the theoretical calculation and simulation methods were adopted to completely study the natural frequency variation under large stroke variation of attachment stiffness.

\section{Simulation-based validation of the new dynamic model}

In order to validate the dynamic model developed in this paper, the dynamic equations from Eq.(1) to Eq.(15) are utilized to calculate the natural frequencies, which are firstly compared with a set of results obtained simulations. Without loss of generality, the results at the following configurations were utilized for the comparison:

a. Symmetrical leg layout: two adjacent feet are contacted together and symmetrically scattered on the same diameter of a circle (Figure 6 (a));

b. Arbitrary leg layout: the six feet arbitrarily scattered on the circles with different diameters (Figure 6 (b));

Two cases were studied by utilizing these two configurations. One case was to study the influence of the natural frequency from the variable attachment stiffness; the other one was to study the difference of natural frequency by considering the leg mass.

\subsection{Setups of Free-Hex}

Firstly, the configuration utilized for validating the dynamic model is introduced in this part. As shown in Figure 5, the generic geometrical and dynamic characteristics of Free-Hex are presented. As an example, the dynamic parameters of one leg (e.g. mass, moment of inertia and length with the joints in leg 4) are specified here, while the other five legs share the same dynamic parameters with leg 4 . 


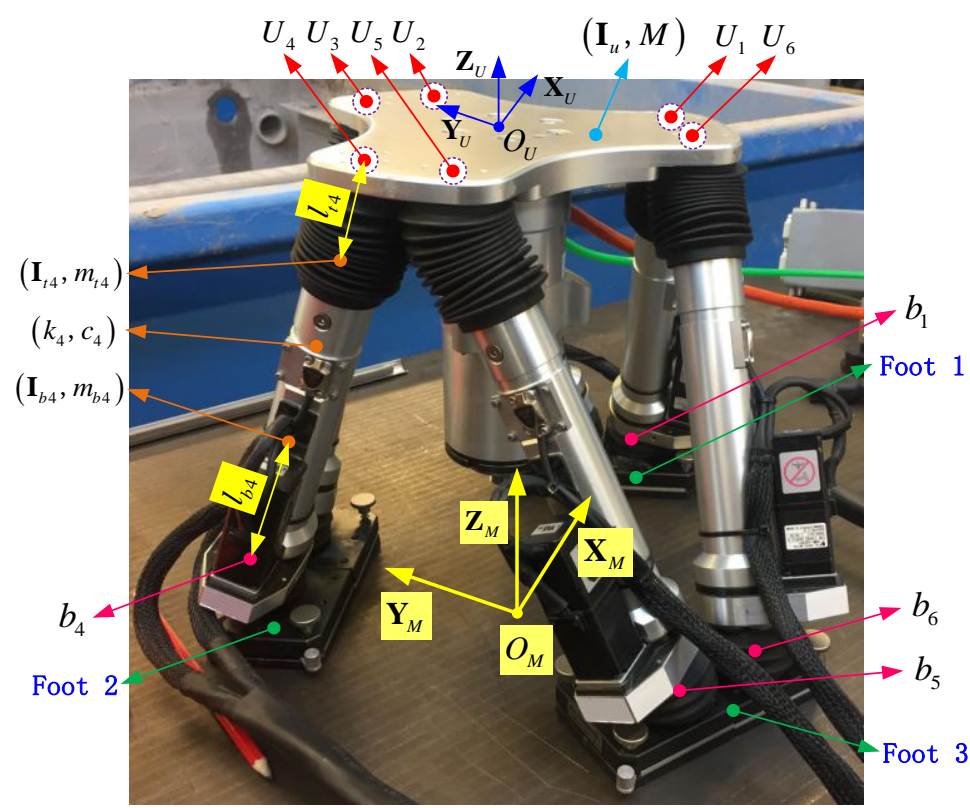

Figure 5. Geometrical and dynamic parameters overlaid on the Free-Hex

The parameters of the Free-Hex for calculating the natural frequencies are listed in Table.1. By substituting these parameters into the dynamic vibration Eq.(17), the natural frequencies in the specific machine configuration can be calculated.

Table.1 Parameters of the machine for dynamic modelling

\begin{tabular}{|c|c|c|c|}
\hline Variables & Variable description & Value & Unit \\
\hline $\boldsymbol{b}_{1}$ & The coordinate of lower spherical joint $b_{1}$ (in $\{M\}$ ) & {$[-250,-35,20]$} & $\mathrm{mm}$ \\
\hline $\boldsymbol{b}_{2}$ & The coordinate of lower spherical joint $b_{2}$ (in $\{M\}$ ) & {$[250,35,20]$} & $\mathrm{mm}$ \\
\hline $\boldsymbol{b}_{3}$ & The coordinate of lower spherical joint $b_{3}$ (in $\{M\}$ ) & {$[-95,234,20]$} & $\mathrm{mm}$ \\
\hline $\boldsymbol{b}_{4}$ & The coordinate of lower spherical joint $b_{4}$ (in $\{M\}$ ) & {$[-155,199,20]$} & $\mathrm{mm}$ \\
\hline $\boldsymbol{b}_{5}$ & The coordinate of lower spherical joint $b_{5}$ (in $\{M\}$ ) & {$[-155,-199,20]$} & $\mathrm{mm}$ \\
\hline $\boldsymbol{b}_{6}$ & The coordinate of lower spherical joint $b_{6}$ (in $\{M\}$ ) & {$[-95,-234,20]$} & $\mathrm{mm}$ \\
\hline $\boldsymbol{u}_{1}$ & The coordinate of upper spherical joint $u_{1}$ (in $\{U\}$ ) & {$[64,-70,0]$} & $\mathrm{mm}$ \\
\hline $\boldsymbol{u}_{2}$ & The coordinate of upper spherical joint $u_{2}$ (in $\{U\}$ ) & {$[64,70,0]$} & $\mathrm{mm}$ \\
\hline $\boldsymbol{u}_{3}$ & The coordinate of upper spherical joint $u_{3}$ (in $\{U\}$ ) & {$[28,91,0]$} & $\mathrm{mm}$ \\
\hline $\boldsymbol{u}_{4}$ & The coordinate of upper spherical joint $u_{4}$ (in $\{U\}$ ) & {$[-93,20,0]$} & $\mathrm{mm}$ \\
\hline $\boldsymbol{u}_{5}$ & The coordinate of upper spherical joint $u_{5}($ in $\{U\})$ & {$[-93,-21,0]$} & $\mathrm{mm}$ \\
\hline $\boldsymbol{u}_{6}$ & The coordinate of upper spherical joint $u_{6}$ (in $\{U\}$ ) & {$[29,-91,0]$} & $\mathrm{mm}$ \\
\hline $\boldsymbol{I}_{u}$ & The moment inertia of upper platform & Diag. $(1.68 \mathrm{e}+2,1.68 \mathrm{e}+2,7.26 \mathrm{e}+3)$ & Kg. $\mathrm{m}^{2}$ \\
\hline$k_{i}$ & The stiffness of one leg $(i=1, \ldots, 6)$ & $1.35 \mathrm{e}+6$ & $\mathrm{~N} / \mathrm{m}$ \\
\hline$m$ & Mass of upper platform & 2.8 & $\mathrm{Kg}$ \\
\hline$l_{b i}$ & $\begin{array}{l}\text { Distance from the lower leg part mass centre to lower } \\
\text { spherical joint point }(i=1, \ldots, 6)\end{array}$ & 100 & $\mathrm{~mm}$ \\
\hline$l_{t i}$ & $\begin{array}{l}\text { Distance from the upper leg part mass centre to upper } \\
\text { spherical joint point }(i=1, \ldots, 6)\end{array}$ & 87 & $\mathrm{~mm}$ \\
\hline $\boldsymbol{I}_{b i}$ & Moment of inertia of lower leg part $(i=1, \ldots, 6)$ & Diag. (1.7e-4, 2.4e-6,1.7e-4) & Kg. $\mathrm{m}^{2}$ \\
\hline $\boldsymbol{I}_{t i}$ & Moment of inertia of upper leg part $(i=1, \ldots, 6)$ & Diag. (1.3e-3, 1e-4, 1.4e-4) & Kg. $\mathrm{m}^{2}$ \\
\hline
\end{tabular}

Note: $\{M\}$ and $\{U\}$ are the coordinate systems of the machine tool and upper platform respectively. 
Since the six feet of the Free-Hex can be set/clamped on the contacting surface individually, two of the most representative system configurations (i.e. symmetrical and arbitrary) are chosen to be analyzed to validate the proposed dynamic model, as seen in Figure 6. Further, the coordinates and orientations of the six feet in these two configurations are presented in Table.2. After calibrated the configuration of Free-Hex [29,30], the simulation and validation can proceed.

Table.2 Foot coordinates and angles of the Free-Hex in different configurations

\begin{tabular}{ccccc}
\hline Configuration & \multicolumn{2}{c}{$1($ In Figure 6 (a)) } & \multicolumn{2}{c}{$2($ In Figure 6 (b)) } \\
\hline Type & Position (mm) & Angle (Deg) & Position (mm) & Angle (Deg) \\
Foot 1 & $(250,-35,0)$ & -90 & $(170,0,0)$ & -90 \\
Foot 2 & $(250,35,0)$ & $($ as foot 1$)$ & $(125,217,0)$ & -90 \\
Foot 3 & $(-95,234,0)$ & 30 & $(-85,-147,0)$ & 30 \\
Foot 4 & $(-155,199,0)$ & (as foot 2) & $(-250,0,0)$ & 90 \\
Foot 5 & $(-155,-199,0)$ & 150 & $(-85,-147,0)$ & 150 \\
Foot 6 & $(-95,-234,0)$ & (as foot 3) & $(125,-217,0)$ & 210 \\
\hline
\end{tabular}

In Figure 6 (a), every two adjacent feet are connected together and symmetrically spaced on a circle, which is the most widely utilized configuration due to its high stiffness. As aforementioned, in some scenarios, the three groups of feet (two adjacent feet constituted as one group) have to be placed on unequal stiffness surfaces to fulfill the repair/maintenance task. Hence, one group (Foot 1 in Figure 6 (a)) is located on the surface with finite stiffness $\left(k_{s}\right)$, while the other two groups of feet are fully fixed on the surfaces with infinite stiffness. As a comparison, in Figure 6 (b), the six feet are arbitrarily placed, and one foot (Foot 1 in Figure 6 (b)) is located on the surface with finite stiffness $\left(k_{s}\right)$, while the other five feet are fully fixed on surfaces with infinite stiffness.

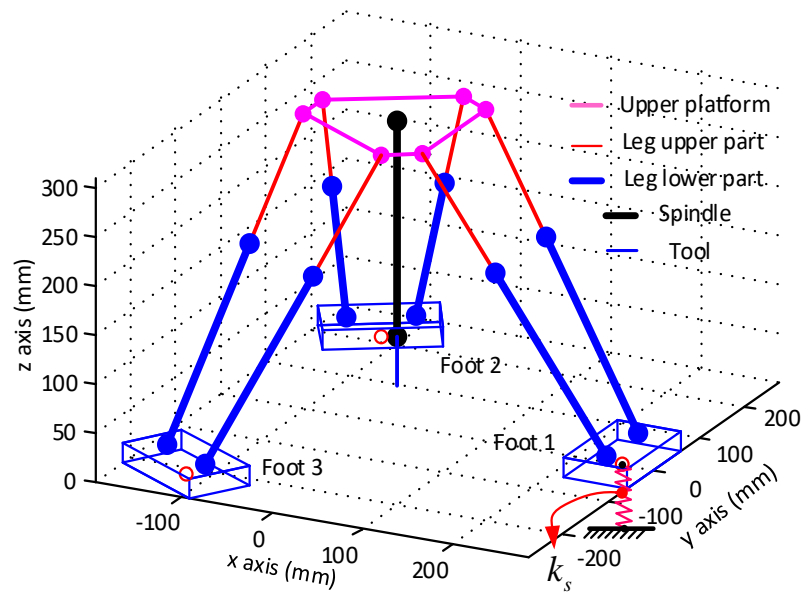

a

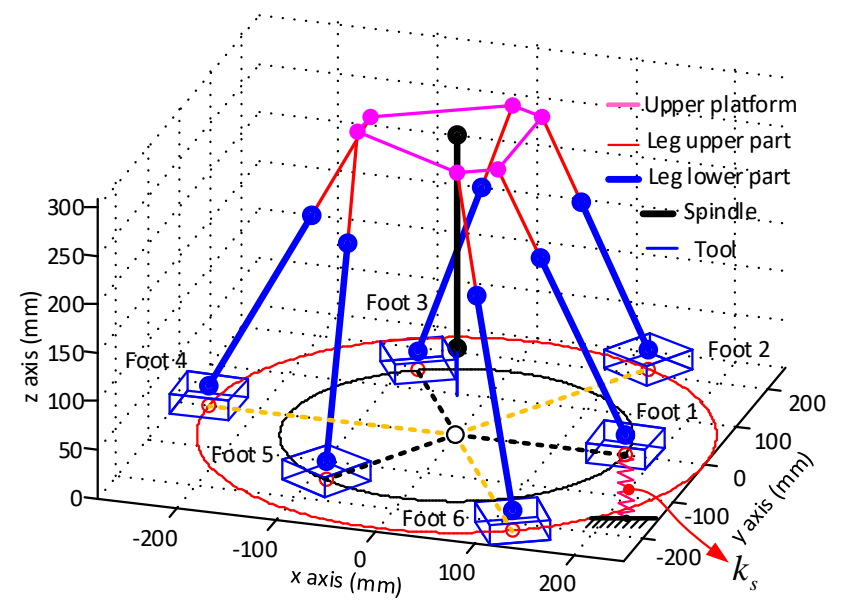

b

Figure 6. Free-Hex machine in two setups: a) symmetrical configuration: the two adjacent feet are contacted together and symmetrically scattered on the same circle; foot 1 is located on the surface with variable stiffness while the other two feet are fully fixed on the ground. b) Arbitrary configuration: the six feet arbitrarily scattered on circles with different diameters; foot 1 is located on the surface with variable stiffness while the other five feet are fully fixed on the ground

Further, the equation developed in this paper was utilized to obtain the difference of natural frequencies of the Free-Hex with the variation of attachment stiffness. In order to validate the calculation, the CAD model of the FreeHex at these two configurations are imported into ANSYS individually for computing the first six natural frequencies 
of the system.

\subsection{Physical prototype-based validation}

Followed by the simulation validation, the physical prototype of the Free-Hex was utilized for cross-checking the results obtained in Chapter 4. Impact ('hammer') testing equipment is used to obtain the natural frequencies of the Free-Hex prototype, and the test results are compared with the corresponding theoretical calculation results. The model parameters used in this paper are those associated with the prototypes of the first generation Free-Hex (Figure 5). The six feet are fixed on the experimental table to execute the impact test in two configurations (i.e. symmetrical and arbitrary) as seen in Figure 6.

In order to modify the attachment stiffness, a mechanism that can adjust the stiffness was design and constructed. Figure 7 illustrates the mechanism proposed in this paper for adjusting the stiffness of the foot connection. The lower platform of the stiffness variation mechanism is fixed on the experimental table (its stiffness can be considered as infinite) with the bolts, and the foot of the Free-Hex can be fixed on the upper platform of the stiffness variation mechanism using the magnetic chuck. In order to realize the bidirectional vibrations of the upper platform, two springs with equal stiffness are installed between the upper and lower platforms. When a force is applied on the stiffness variation mechanism, the two springs will have the same length compression deformation, which supports the pair of the feet with a finite stiffness. Further, by replacing the springs with different stiffness constants, the stiffness of the entire mechanism can be adjusted. The main bolt (i.e. adjust bolt in Figure 7) and shaft head are used to pre-tension the spring so that, the foot is damped in both directions (i.e. up and downwards).

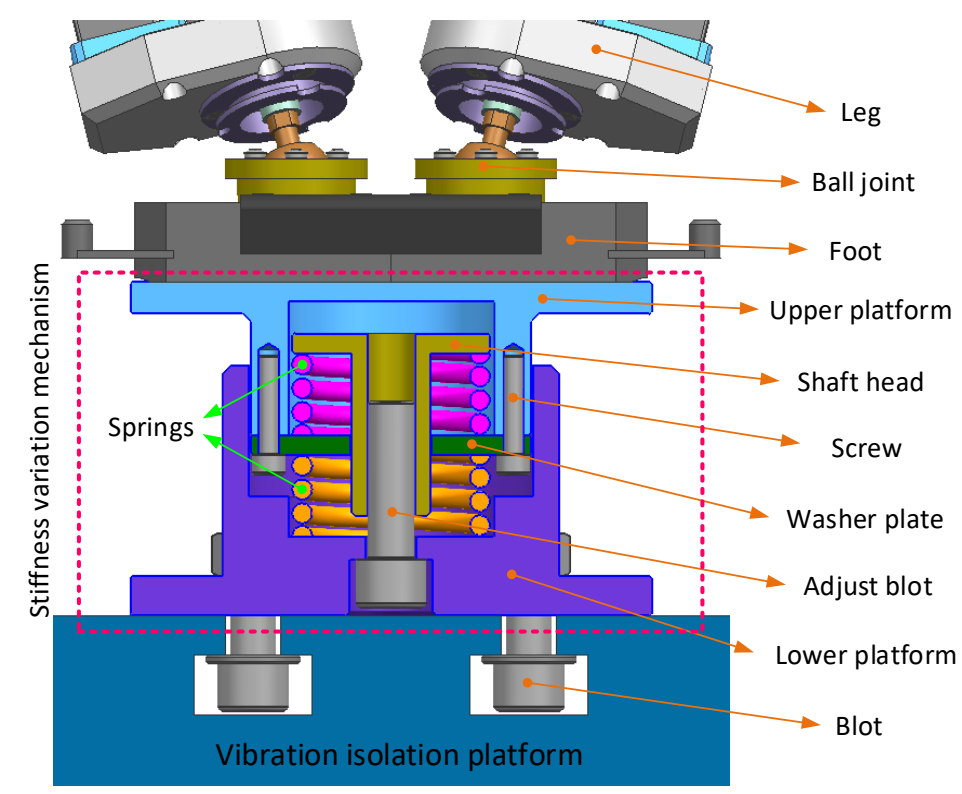

Figure 7. 3D structure of the stiffness variation mechanism to simulate the unequal stiffness of the attachment surfaces.

As shown in Table.3, a series of springs with suitable sizes are chosen with the stiffness varying from $7.3 \mathrm{e}+4$ $\mathrm{N} / \mathrm{m}$ to $4.13 \mathrm{e}+5 \mathrm{~N} / \mathrm{m}$ for the experiment test. For each experiment test, one of the springs is installed into the stiffness variation mechanism, and then the machine is configured. After that, the impact testing device is used to get the vibration response of the Free-Hex.

Table.3 Parameters of the springs for experiments 


\begin{tabular}{cccccccccc}
\hline Number & 1 & 2 & 3 & 4 & 5 & 6 & 7 & 8 & 9 \\
\hline Stiffness (N/m) & $7.3 \mathrm{e}+4$ & $1.17 \mathrm{e}+5$ & $1.54 \mathrm{e}+5$ & $1.92 \mathrm{e}+5$ & $2.15 \mathrm{e}+5$ & $2.45 \mathrm{e}+5$ & $2.88 \mathrm{e}+5$ & $3.39 \mathrm{e}+5$ & $4.13 \mathrm{e}+5$ \\
\hline
\end{tabular}

The feet of the Free-Hex are fixed on the experimental table to acquire the natural frequencies in the first six modes at the two configurations (Figure 6), separately. The stiffness variation mechanism is used to adjust the attachment stiffness of the Free-Hex, and then the impact testing is implemented, and the corresponding vibration measurements are collected by the data logger to calculate the frequency spectrums. As the Free-Hex has 6 DOFs, the impacts are imposed by the hammer in six directions (three along the $\boldsymbol{X}, \boldsymbol{Y}$ and $\boldsymbol{Z}$ axes and three around the $\boldsymbol{X}$, $\boldsymbol{Y}$ and $\boldsymbol{Z}$ axes).

In Figure 8 (a), two adjacent feet are connected together as one foot (three groups of feet at this configuration), and one group of the feet are located on the stiffness variation mechanism (Figure 6 (a)). In Figure 8 (b), the FreeHex is in an arbitrary configuration and one foot is located on the stiffness variation mechanism (Figure 6 (b)).

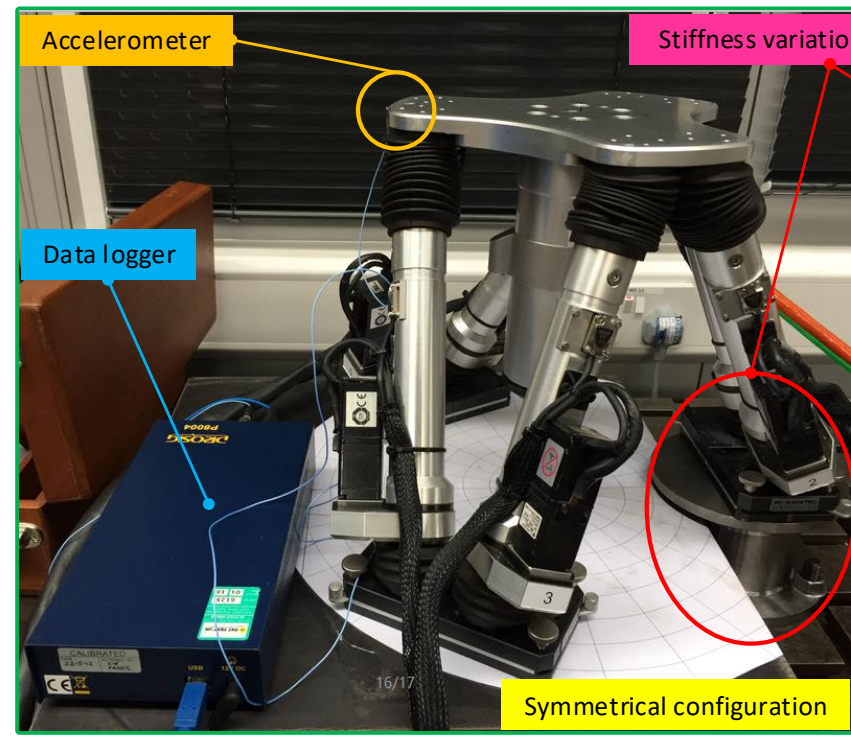

(a)

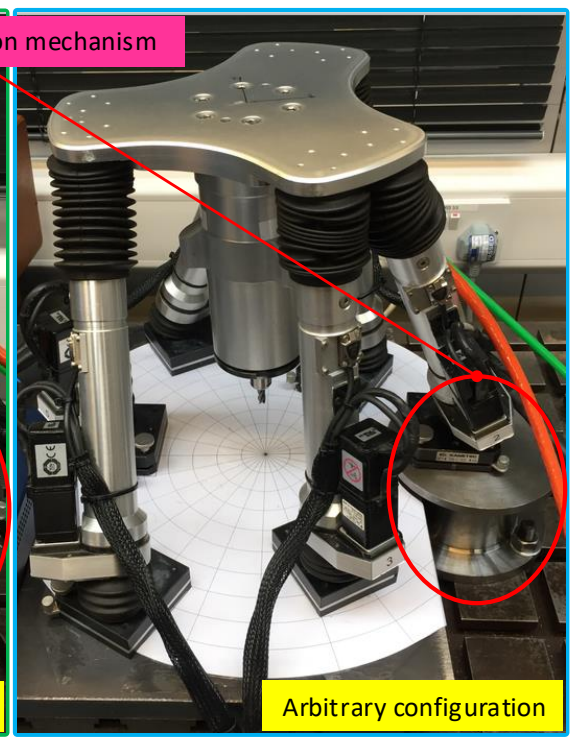

(b)

Figure 8. Modal test of the Free-Hex for the two configurations using the stiffness variation mechanism. a) symmetrical configuration: the two adjacent feet are contacted together as one foot, and one of feet is configured on the stiffness variation mechanism. b) arbitrary configuration: the six feet arbitrarily scattered on circles with different diameters, and one of the foot is configured on the stiffness variation mechanism. (Note: two feet as one group are located on the stiffness variation mechanism in a); only one foot is located on the stiffness variation mechanism in b))

The modal test equipment used in this experiment is DATS_P8004, which has four analogue single input channels and a one-speed input channel. In this modal test, one accelerometer was used to acquire the vibration signals of the Free-Hex in $\boldsymbol{X}, \boldsymbol{Y}, \boldsymbol{Z}$ axes, respectively, and a hammer with an elastic tip was used to excite the machine.

The positions of feet that are located on the steel pads and on the stiffness variation mechanism at the two machine configurations are the same as Table.2. The springs with different stiffness (Table.3) are used in turn to change the attachment stiffness, and the impact testing experiment is implemented to get the corresponding natural frequencies. Then the attachment stiffness (Table.3) is substituted into the theoretical derivation presented in this paper to calculate the natural frequencies, and the kinematic and dynamic parameters of the machine are used as Table.1. The comparison between the experimental results and theoretical calculations is shown in Figure 10. 


\begin{tabular}{|cccccc}
\hline $\begin{array}{c}\text { Modal frequencies } \\
\text { Experimental }\end{array}$ & $1^{\text {st }}$ & $2^{\text {nd }}$ & $3^{\text {rd }}$ & $4^{\text {th }}$ & $6^{\text {th }}$ \\
Calculation & $*^{\text {th }}$ & + & $\checkmark$ & $\Delta$ \\
\hline
\end{tabular}

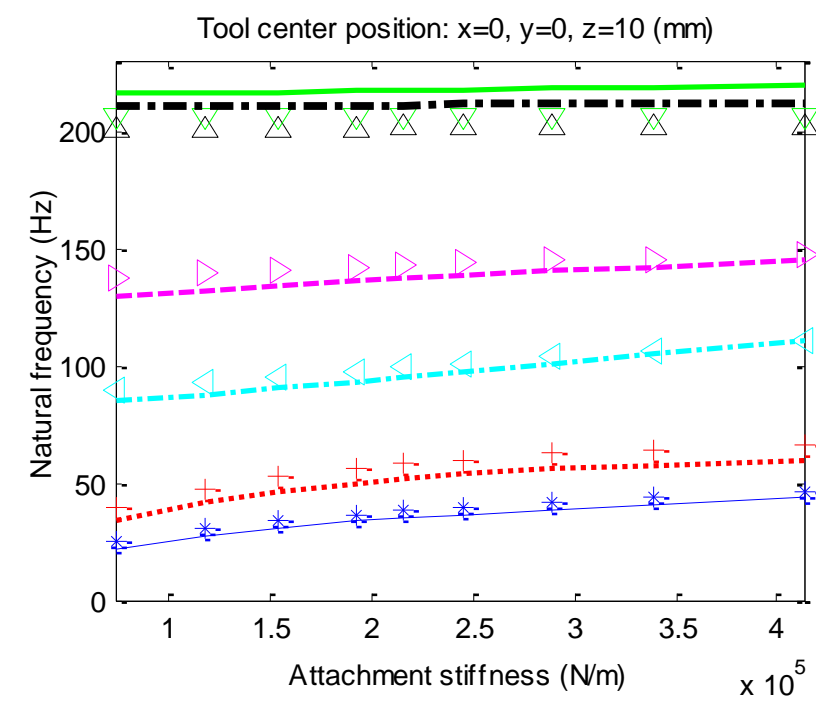

(a)

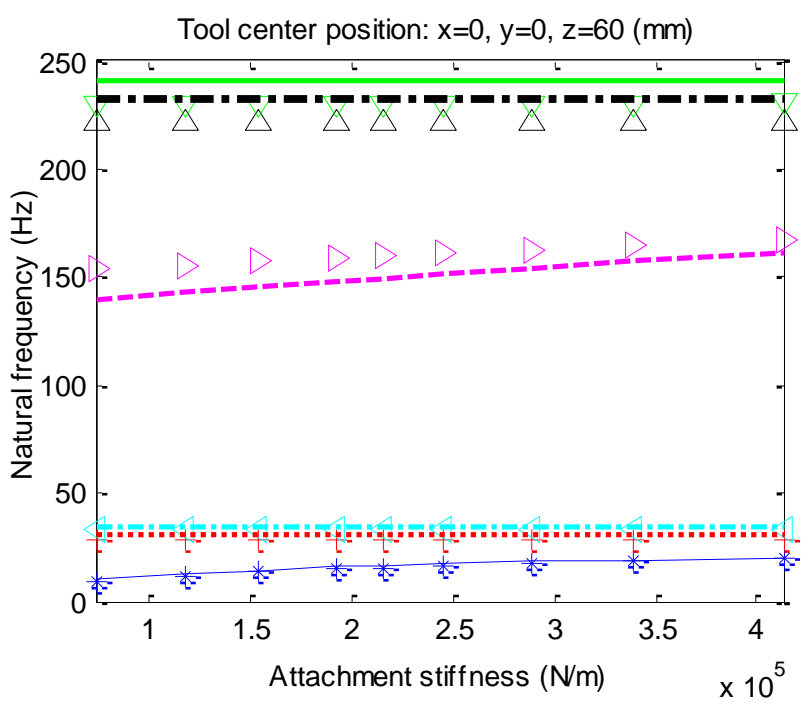

(b)

Figure 9. Comparison between the theoretical calculation and experimental tests for: a) symmetrical and b) arbitrary configurations of the feet of the Free-Hex on the base surface

Therefore, it can be concluded that the experimental results match well with the calculation from the dynamic model at the test configurations (Figure 6) and the natural frequencies of the Free-Hex are affected by the attachment stiffness at the given machine configurations (i.e. symmetric and arbitrary). Further, it also can be found that the absolute value of the disparity between the experimental and theoretical results in high orders (e.g., $5^{\text {th }}$ and $6^{\text {th }}$ ) are greater than that of the lower orders (e.g., $1^{\text {st }}$ and $2^{\text {nd }}$ ). But, the percentage of the disparities over the experimental results remain constantly under $6 \%$, which are $5.5 \%, 5.2 \%, 4.3 \%, 4.4 \%, 4.9 \%$ and $5.4 \%$ in the six modes, respectively.

\subsection{Model-based dynamic analysis of Free-Hex with unequal attachment stiffness}

In order to validate the dynamic model of the Free-Hex, the aforementioned configurations (Figure 6 (a)\&(b)) are employed for the case study to understand the influence of the machine natural frequencies from the stiffness of the attachment. Specifically, the attachment stiffness is changed gradually to research the variation of the natural frequencies under two machine configurations (i.e. symmetrical and arbitrary). Further, the natural frequencies of Free-Hex with and without considering the leg masses are calculated and compared with FEA simulation results.

In this section, the influence of the attachment stiffness on the natural frequencies is studied with two configurations separately. At each configuration, the stiffness of the attached surface is varied from $10^{0} \mathrm{~N} / \mathrm{m}$ to $10^{12}$ $\mathrm{N} / \mathrm{m}$ to analyze the frequency variation; since $10^{12} \mathrm{~N} / \mathrm{m}$ is significantly greater than the stiffness of each leg, this value is chosen as the end condition (which is close to infinite stiffness). The natural frequencies are calculated using the dynamic equation presented in Chapter 3 and simulated in ANSYS software to validate the theoretical formulae developed in this paper.

Figure 10 illustrates the natural frequency variations of Free-Hex with the change of attachment stiffness in the connection. Also, the leg mass was included in the calculation and simulation. Specifically, Figure 10 (a) shows the frequencies of the machine at the symmetrical configuration (Figure 6 (a)), while Figure 10 (b) presents the frequencies of the machine in the arbitrary configuration (Figure 6 (b)). In these two figures, the lines represent the 
results obtained from the theoretical model developed in this paper, while the FEA results are denoted as the points. The tool center position is chosen to scale the structural configuration of the Free-Hex during the machining for the dynamic analysis since the workspace is calculated and plotted basing on the position of the tool center. The machine is regarded to have no rotational motion of the upper platform, which simplifies the validation process. The parameters of the machine are listed in Table.1, while the feet positions and angles are listed in Table.2.

\begin{tabular}{|ccccccc|}
\hline Modal frequencies & $1^{\text {st }}$ & $2^{\text {nd }}$ & $3^{\text {rd }}$ & $4^{\text {th }}$ & $5^{\text {th }}$ & $6^{\text {th }}$ \\
Simulation & $*^{*}$ & + & $\triangleleft$ & $\triangleright$ & $\Delta$ & $\nabla$ \\
Calculation & & $\cdots$ & - & - & - & - \\
\hline
\end{tabular}

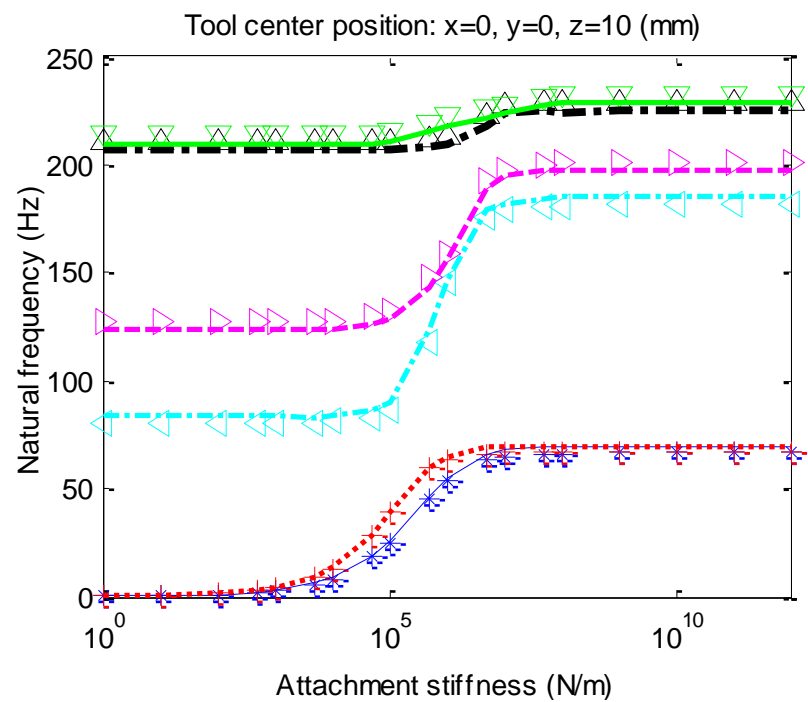

(a)

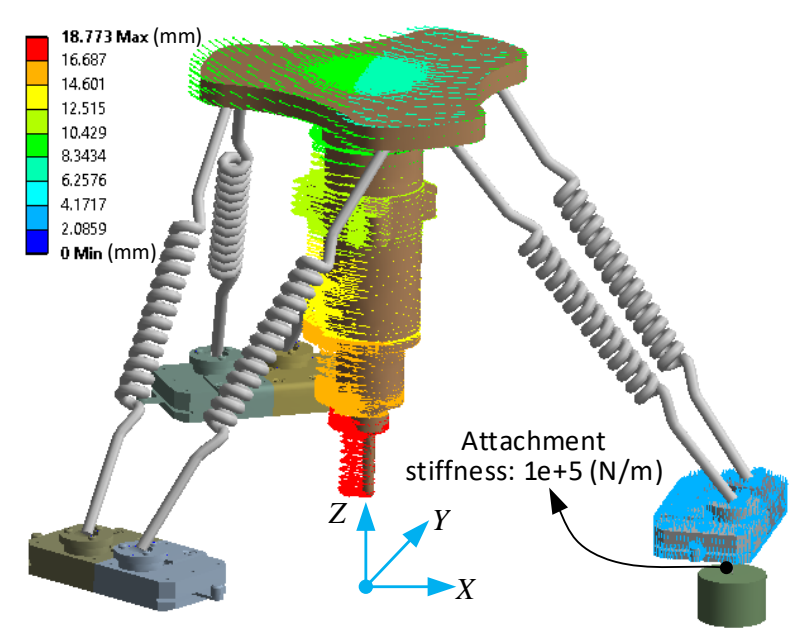

(c)

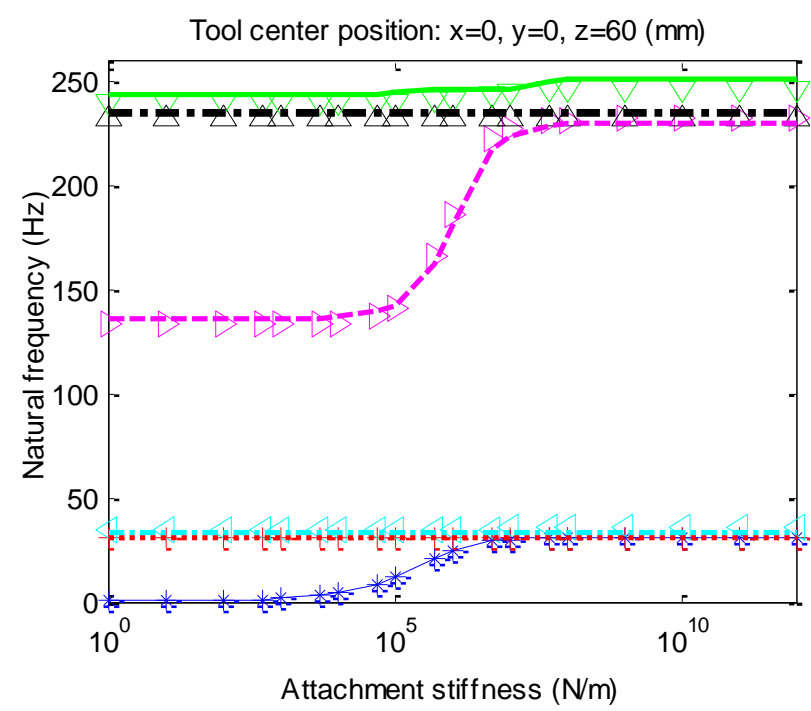

(b)

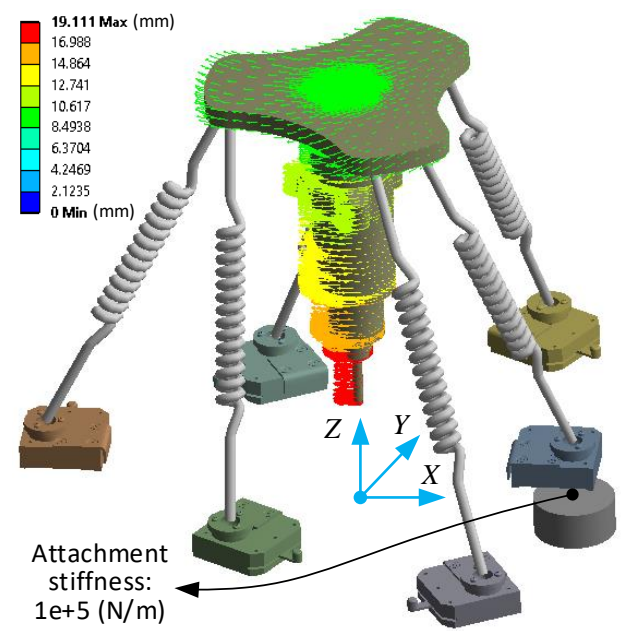

(d)

Figure 10. Natural frequencies versus stiffness in the connection. a) Symmetrical configuration; b) Arbitrary configuration; c) and d) are the examples of mode shapes of $1^{\text {st }}$ mode under specified attachment stiffness in symmetrical and arbitrary configurations of Free-

Hex respectively. (Note: the centre of the work volume was selected as the tool centre position since the size and shape of work volume of Free-Hex changes a lot under different configurations)

In Figure 10 (a), it can be seen that all the six modal frequencies rise when the attachment stiffness was increased, and the frequencies at $10^{12} \mathrm{~N} / \mathrm{m}$ are close to the results calculated when the feet fully attached to the surface with infinite stiffness. The greatest variation of the frequency $(95 \mathrm{~Hz})$ occurs at the $3^{\text {rd }}$ mode, and the modal frequencies 
of the $4^{\text {th }}, 1^{\text {st }}$ and $2^{\text {nd }}$ modes increase by $65 \mathrm{~Hz}, 50 \mathrm{~Hz}$ and $50 \mathrm{~Hz}$ respectively, while the last two modal frequencies (i.e. the $5^{\text {th }}$ and $6^{\text {th }}$ modes) have the least influence from the attachment stiffness variation, about $17 \mathrm{~Hz}$ and $15 \mathrm{~Hz}$ respectively.

While in Figure 10 (b), the frequency variations among the six modes of the Free-Hex in the arbitrary configuration are much different from the symmetrical configuration. Only three modal frequencies (i.e. the $1^{\text {st }}, 4^{\text {th }}$ and $6^{\text {th }}$ modes) are affected by the attachment stiffness, and the rest of the modal frequencies are irrelevant with the attachment stiffness. The influence among the three affected modal frequencies are $85 \mathrm{~Hz}\left(4^{\text {th }}\right), 40 \mathrm{~Hz}\left(1^{\text {st }}\right)$ and 13 $\mathrm{Hz}\left(6^{\text {th }}\right)$ respectively.

An analysis of the mode shapes revealed the following: 1. For understanding the vibration directions of FreeHex under different configurations (i.e. symmetrical and arbitrary respectively) with specified attachment stiffness (i.e. $10^{5} \mathrm{~N} / \mathrm{m}$ ), the examples of mode shapes under $1^{\text {st }}$ mode were displayed to discover how the machine configuration influences the mode shape, seen in Figure 10 (c) and (d). It can be found from results that the vibration directions under two configurations are closing to the translation motion along $\boldsymbol{X}$ axis; 2. For the different attachment stiffness, the overall stiffness matrix, $\boldsymbol{K}$, will be changed accordingly, which may lead to the change of eigenvector (mode shape) in Eq.(17). In this paper, the focus was devoted to the natural frequency variation analysis of Free-Hex under different attachment stiffness, which is more important to model the vibrations during machining. For the influence of attachment stiffness on the mode shape, it is beyond the scope of this paper, but will be studied in the future. Thus, only the mode shapes of Free-Hex under a given attachment stiffness were displayed for briefly understanding the vibration directions in different modes of natural frequency. The detailed relationship will be researched in future studies.

Comparing the results (i.e. modal frequencies) obtained from Figure 10 (a) and (b), it can be seen that the natural frequencies under two machine configurations varied greatly at the same attachment stiffness, which indicates that the natural frequencies are greatly influenced by attachment stiffness. For example, when the attachment stiffness is under $10^{12} \mathrm{~N} / \mathrm{m}$, the modal frequencies of the $2^{\text {nd }}$ and $3^{\text {rd }}$ are $42 \mathrm{~Hz}$ and $89 \mathrm{~Hz}$ in symmetrical configuration, while 27 $\mathrm{Hz}$ and $28 \mathrm{~Hz}$ in the arbitrary configuration.

\section{Conclusion}

The Free-Hex, as a portable machine tool, is able to be attached to a wider range of workpiece geometries, e.g. flat, curved, tilted and even unstructured surfaces. Nevertheless, in some scenarios (e.g. in-situ repair), the limbs of the Free-Hex have to be attached to the surfaces with unequal stiffness due to the space limitations (e.g. in-situ intervention); this brings the challenge of dynamic modelling such complex structure to determinate the machine tool setup to improve the machining performance.

For the first time, the effect of attachment stiffness on natural frequencies was studied on the portable parallel machine tool, which requires the novel dynamic model to be developed (i.e. the variables of the attachment stiffness and limb mass are included in the model). In order to validate the model, several experiments (i.e. impact testing on the Free-Hex machine) and simulation (i.e. FEA) were conducted at different configurations of the machine tool (i.e. the symmetric and arbitrary configurations). The errors between the calculation and physical experiments were about $5.1 \%$ for the symmetrical configuration and $5.8 \%$ for the arbitrary configuration. Compared with the results from the calculation, it was found that the errors between the theoretical calculation and simulation are $3.7 \%$ at the symmetrical configuration and $4.2 \%$ at the arbitrary configuration. It proves that the model developed in this paper can properly represent the dynamic characteristics of the Free-Hex when the limbs are attached to surfaces with unequal stiffness. Hence, the results obtained from this paper (i.e. the natural frequencies are greatly influenced by the environmental attachment stiffness) can be utilized for configuring the limbs of the Free-Hex machine tool in confined space when the system is employed for real industrial in-situ repair interventions. 
Further, by applying the validated dynamic model, the natural frequency variation of Free-Hex under a wider range of attachment stiffness was studied. It was found that the natural frequencies were largely influenced by the machine configuration and attachment stiffness: firstly, for machine configuration on the influence of natural frequency, for example, the modal frequencies of mode 3 at the both test configurations were greatly affected (i.e. $170 \mathrm{~Hz}$ and $40 \mathrm{~Hz}$ at the symmetrical and arbitrary configurations respectively, Figure 10), provided that the attachment stiffness is infinite; then, for attachment stiffness on the influent of natural frequency, for example, the first four modal frequencies (i.e. mode 1 to 4 ) were remarkably increased (by $72 \%$ in average) at the symmetrical configuration, while only two modal frequencies (i.e. modes 1 and 4) are affected (69\% in average) at the arbitrary configuration, when the attachment stiffness of one limb was adjusted from 0 to $1 \mathrm{e}+12 \mathrm{~N} / \mathrm{m}$ and the other limbs were remained as infinite.

\section{Acknowledgment}

The research leading to these results has received funding from China Scholarship Council, University of Nottingham and UK EPSRC (Robotics and Artificial Intelligence for Nuclear - EP/R026084/1).

\section{Reference}

[1] Mohit Law, Yusuf Altintas, A. Srikantha Phani. Rapid evaluation and optimization of machine tools with positiondependent stability[J]. International Journal of Machine Tools and Manufacture. 2013. 68: 81-90

[2] Reimund Neugebauer, M. Wabner, H. Rentzschet al. Structure principles of energy efficient machine tools[J]. CIRP Journal of Manufacturing Science and Technology. 2011. 4 (2) : 136-147

[3] R. Neugebauer, U. Priber, H. Rentzschet al. Mobile systems for machining large work pieces[M]Springer, 2012: 135-140

[4] A. Olarra, J. M. Allen, D. A. Axinte. Experimental evaluation of a special purpose miniature machine tool with parallel kinematics architecture: Free leg hexapod[J]. Precision Engineering. 2014. 38 (3) : 589-604

[5] Adam Rushworth, Salvador Cobos-Guzman, Dragos Axinteet al. Pre-gait analysis using optimal parameters for a walking machine tool based on a free-leg hexapod structure[J]. Robotics and Autonomous Systems. 2015. 70: 3651

[6] J. M. Allen, D. A. Axinte, T. Pringle. Theoretical analysis of a special purpose miniature machine tool with parallel kinematics architecture: free-leg hexapod[J]. Proceedings of the Institution of Mechanical Engineers, Part B: Journal of Engineering Manufacture. 2012. 226 (3) : 412-430

[7] K. H. Harib, AMM Sharif Ullah, A. Hammami. A hexapod-based machine tool with hybrid structure: kinematic analysis and trajectory planning[J]. International Journal of Machine Tools and Manufacture. 2007. 47 (9) : 14261432

[8] M. J. Nategh, M. M. Agheli. A total solution to kinematic calibration of hexapod machine tools with a minimum number of measurement configurations and superior accuracies[J]. International Journal of Machine Tools and Manufacture. 2009. 49 (15) : 1155-1164

[9] Orkun O Z C S Ahin, Alper Ert U Rk, H. Nevzat O Zg U Venet al. A closed-form approach for identification of dynamical contact parameters in spindle--holder--tool assemblies[J]. International Journal of Machine Tools and Manufacture. 2009. 49 (1) : 25-35

[10] Congying Deng, Jianguo Miao, Bo Weiet al. Evaluation of machine tools with position-dependent milling stability based on Kriging model[J]. International Journal of Machine Tools and Manufacture. 2018. 124: 33-42 
[11] Bin Li, Bo Luo, Xinyong Maoet al. A new approach to identifying the dynamic behavior of CNC machine tools with respect to different worktable feed speeds[J]. International Journal of Machine Tools and Manufacture. 2013. 72: $73-84$

[12] Mohit Law, Hendrik Rentzsch, Steffen Ihlenfeldt. Predicting mobile machine tool dynamics by experimental dynamic substructuring[J]. International Journal of Machine Tools and Manufacture. 2016. 108: 127-134

[13] K. Harib, K. Srinivasan. Kinematic and dynamic analysis of Stewart platform-based machine tool structures[J]. Robotica. 2003. 21 (5): 541

[14] Parthajit Mukherjee, Bhaskar Dasgupta, A. K. Mallik. Dynamic stability index and vibration analysis of a flexible Stewart platform[J]. Journal of sound and vibration. 2007.307 (3) : 495-512

[15] Jiao Mo, Zhu-Feng Shao, Liwen Guanet al. Dynamic performance analysis of the X4 high-speed pick-and-place parallel robot[J]. Robotics and Computer-Integrated Manufacturing. 2017. 46: 48-57

[16] Xiulong Chen, Liangkai Wu, Yu Denget al. Dynamic response analysis and chaos identification of 4-UPS-UPU flexible spatial parallel mechanism[J]. Nonlinear Dynamics. 2017. 87 (4) : 2311-2324

[17] Imed Zaghbani, Victor Songmene. Estimation of machine-tool dynamic parameters during machining operation through operational modal analysis[J]. International Journal of Machine Tools and Manufacture. 2009. 49 (12-13): 947-957

[18] M. Postel, O. O Zsahin, Y. Altintas. High speed tooltip FRF predictions of arbitrary tool-holder combinations based on operational spindle identification[J]. International Journal of Machine Tools and Manufacture. 2018. 129: 48-60

[19] P. Albertelli, M. Goletti, M. Monno. A new receptance coupling substructure analysis methodology to improve chatter free cutting conditions prediction[J]. International Journal of Machine Tools and Manufacture. 2013. 72: 1624

[20] H. B. Guo, H. R. Li. Dynamic analysis and simulation of a six degree of freedom Stewart platform manipulator[J]. Proceedings of the Institution of Mechanical Engineers, Part C: Journal of Mechanical Engineering Science. 2006.

220 (1) : 61-72

[21] Siamak Pedrammehr, Mehran Mahboubkhah, Navid Khani. Improved dynamic equations for the generally configured Stewart platform manipulator[J]. Journal of mechanical science and technology. 2012. 26（3）:711-721

[22] H. Z. Jiang, J. F. He, Z. Z. Tong. Modal space control for a hydraulically driven Stewart platform[J]. Journal of Control Engineering and Technology. 2012. 2 (3) : 106-115

[23] Yangmin Li, Qingsong Xu. Stiffness analysis for a 3-PUU parallel kinematic machine[J]. Mechanism and machine theory. 2008. 43 (2) : 186-200

[24] Xiaohui Han, Yuhan Wang, Jing Shi. Stiffness Modeling of 3-PRS Mechanism[M]World Academy of Science, Engineering and Technology (WASET), 2012: 876

[25] V. T. Portman, V. S. Chapsky, Y. Shneor. Workspace of parallel kinematics machines with minimum stiffness limits: Collinear stiffness value based approach[J]. Mechanism and Machine Theory. 2012. 49: 67-86

[26] Nan Ma, Jingjun Yu, Xin Donget al. Design and stiffness analysis of a class of 2-DoF tendon driven parallel kinematics mechanism[J]. Mechanism and Machine Theory. 2018. 129: 202-217

[27] Behrouz Afzali-Far, Anette Andersson, Kristina Nilssonet al. Influence of strut inertia on the vibrations in initially symmetric Gough--Stewart Platforms - an analytical study[J]. Journal of Sound and Vibration. 2015. 352: 142-157

[28] Behrouz Afzali-Far, Per Lidstr O M, Kristina Nilsson. Parametric damped vibrations of Gough--Stewart platforms for symmetric configurations[J]. Mechanism and Machine Theory. 2014. 80: 52-69

[29] Xiao-Dong Ren, Zu-Ren Feng, Cheng-Ping Su. A new calibration method for parallel kinematics machine tools using orientation constraint[J]. International Journal of Machine Tools and Manufacture. 2009. 49 (9) : 708-721

[30] Y-J Chiu, M-H Perng. Self-calibration of a general hexapod manipulator using cylinder constraints[J]. 
International Journal of Machine Tools and Manufacture. 2003.43（10）: 1051-1066 